\title{
A Computer Simulation Study of the Mesoscopic Structure of the Polyelectrolyte Membrane Nafion
}

\author{
Satoru YAmAмото ${ }^{\dagger}$ and Shi-aki Hyodo \\ Toyota Central R\&D Labs., Inc., Nagakute, Aichi 480-1192, Japan
}

(Received February 14, 2003; Accepted April 18, 2003)

\begin{abstract}
We studied the mesoscopic structure of the perfluorinated sulfonic acid membrane Nafion containing water using a dissipative particle dynamics (DPD) simulation. A Nafion polymer is modeled by connecting coarsegrained particles, which correspond to the hydrophobic backbone of polytetrafluoroethylene and perfluorinated side chains terminated by hydrophilic end particles of sulfonic acid groups. Water is also modeled by the same size particle as adopted in the Nafion model, corresponding to a group of four $\mathrm{H}_{2} \mathrm{O}$ molecules. The Flory-Huggins $\chi$-parameters between DPD particles are estimated from the mixing energy calculation using an atomistic simulation. In the DPD simulation, water particles and hydrophilic particles of Nafion side chains spontaneously form aggregates and are embedded in the hydrophobic phase of the Nafion backbone. This structure is a bicontinuous phase of Nafion and water regions and has a continuous path in the cavity of water in any direction. Although this sponge-like structure is essentially identical to the cluster-network model proposed from the experimental studies, the shape of the water clusters is not spherical but irregular, and the water regions are indistinguishable structures of water clusters and their channels. The cluster size and its dependence on the water content are in good agreement with experimental reports; therefore, the simulated mesoscopic structure is confirmed to be a highly possible one.

KEY WORDS Computer Simulation / Dissipative Particle Dynamics / Polyelectrolyte Membrane / Mesoscopic Structure / Morphology / Nafion Membrane / Water Cluster /
\end{abstract}

The well-known perfluorinated sulfonic acid membranes Nafion are the most common membrane materials used in the polymer electrolyte fuel cell because of their exceptional chemical, thermal and mechanical stability in addition to their reasonable proton conductivity. A Nafion polymer consists of a polytetrafluoroethylene backbone and perfluorinated pendant side chains terminated by sulfonic acid groups. The general molecular structure of Nafion polymer is ${ }^{1}$

$$
\begin{gathered}
-\left[\left(\mathrm{CF}_{2}-\mathrm{CF}_{2}\right)_{n}-\mathrm{CF}-\mathrm{CF}_{2}\right]_{\bar{m}} \\
\mathrm{O}-\mathrm{CF}_{2}-\mathrm{CF}-\mathrm{O}-\mathrm{CF}_{2}-\mathrm{CF}_{2}-\mathrm{SO}_{3} \mathrm{H} \\
\stackrel{\mathrm{C}}{\mathrm{C}} \mathrm{F}_{3}
\end{gathered}
$$

where $n$ is approximately $5-14$, and $m$ is $200-1000$. To investigate the structure and swelling behavior of the hydrated Nafion membranes, numerous experimental efforts have been concentrated on neutron, wide and small angle X-ray scattering, infrared (IR) and Raman spectroscopy, and transmission electron microscopy (TEM) techniques. ${ }^{1-13}$ It has been well established that hydrated Nafion membranes have two phases on a nanometer scale, a hydrophobic phase containing the backbone and a hydrophilic phase containing sulfonic acid groups and water. Several models for these structures such as the interconnected spherical water clusters (the cluster-network model) have been proposed for the interpretation of the scattering patterns. ${ }^{1-7}$ However, these models are still under discussion concerning the size and shape of the water clusters. It is meaningful to clarify the structure of the membranes for analytical study and to improve the mechanical and transport properties of the materials.

In recent years, mesoscopic simulation techniques such as a dynamic mean field theory ${ }^{14,15}$ and a dissipative particle dynamics (DPD) ${ }^{16-21}$ have been applied to study the modeling morphology evolution in polymer systems during phase separation. In these methods, the polymer chain is commonly treated as a coarse-grained bead-spring model. Each bead corresponds to a group of several atoms. Mesoscopic simulation can treat a wide range of length and time scales by many orders of magnitude compared to the atomistic simulations such as molecular dynamics. These mesoscopic simulation methods have been successfully applied to some cases of practical interest such as to the microphase separation of binary polymer mixtures and diblock copolymer systems. ${ }^{14,19,20}$ Although most of the previous applications of the mesoscopic simulation are demonstrations for polymer systems with model parameters, it is applicable to a real system when a simulation parameter such as the Flory-Huggins $\chi$-parameter for a coarse-grained model is determined for actual materials. ${ }^{22,23}$ Several available methods for evaluating the $\chi$ parameter for polymer-polymer, polymer-solvent, and

To whom correspondence should be addressed (Phone: +81-561-63-4693, Fax: +81-561-63-4698, E-mail: e0857@mosk.tytlabs.co.jp). 
solvent-solvent pairs in blend systems have already been proposed using an atomistic simulation based on the cohesive energy or the heat of mixing theory. ${ }^{24-29}$ According to the synthesis process of the Nafion membrane in the case of a casting procedure, ${ }^{13,30}$ the structure of the hydrated Nafion membrane seems to be spontaneously formed from solution in an alcohol and water mixture, in a way similar to the microphase separation of polymer mixture systems. Therefore, it is expected to predict the structure of the hydrated Nafion membranes by a mesoscopic simulation when we use appropriate $\chi$-parameters for the Nafion polymer and water.

In this study, we perform a mesoscopic simulation to study the structure of the hydrated Nafion membrane using an originally coded DPD program. A Nafion polymer molecule is modeled by connecting soft spherical particles, which represent a group of several atoms. Water is also modeled by the same size particle as adopted in the Nafion polymer model, corresponding to a group of several $\mathrm{H}_{2} \mathrm{O}$ molecules. The Flory-Huggins $\chi$-parameters for the different types of particles of Nafion and water models are calculated by an atomistic simulation technique. The mixing energies are calculated for a chemical structure equivalent to each component of DPD particles using molecular simulations by a Monte Carlo approach for pure components and their blends, and then the $\chi$-parameters are estimated. By this procedure, we can perform mesoscopic simulation based on actual material parameters. Simulated structure and dependence on water content of the hydrated Nafion membranes are compared to previous experiments in the literature for verification.

\section{SIMULATION METHOD}

\section{DPD Simulation Method}

The dissipative particle dynamics (DPD) is a relatively new method proposed to study hydrodynamic behavior of complex fluids. ${ }^{16,17}$ The method is based on the dynamics of soft particles interacting by conservative, dissipative, and random forces. By introducing a bead-spring type particle model, the method is extended to polymer systems. ${ }^{19-21}$ Here we describe an outline of the model and the evolution algorithm of the DPD. Now, we consider that the particles are subject to conservative, dissipative, and random forces, and spring forces for connecting spheres. The time evolution of the system is obtained by solving Newton's equation of motion,

$$
\frac{d \boldsymbol{r}_{i}}{d t}=\boldsymbol{v}_{i}
$$

$$
m_{i} \frac{d \boldsymbol{v}_{i}}{d t}=\boldsymbol{f}_{i}
$$

where $\boldsymbol{r}_{i}, \boldsymbol{v}_{i}$, and $m_{i}$ are the position, velocity, and mass of the $i$-th particle, respectively. The force $f_{i}$ contains three parts of the original DPD formula and an additional spring force for the polymer system. The interaction between two particles can be written as the sum of these forces, where the suffixes $C, D, R$, and $S$ represent the conservative, dissipative, random, and spring forces, respectively.

$$
\boldsymbol{f}_{i}=\sum_{j \neq i}\left(\boldsymbol{F}_{i j}^{C}+\boldsymbol{F}_{i j}^{D}+\boldsymbol{F}_{i j}^{R}+\boldsymbol{F}_{i j}^{S}\right)
$$

The first three forces of the original DPD are considered within a certain cutoff radius $r_{c}$. The conservative force $\boldsymbol{F}_{i j}^{C}$ is a soft repulsion acting along the line of centers and is given by ${ }^{19}$

$$
\boldsymbol{F}_{i j}^{C}=\left\{\begin{array}{cc}
-a_{i j}\left(r_{c}-r_{i j}\right) \boldsymbol{n}_{i j} & r_{i j}<r_{c} \\
0 & r_{i j} \geq r_{c}
\end{array}\right.
$$

where $a_{i j}$ is a maximum repulsion force between particle $i$ and $j, \boldsymbol{r}_{i j}=\boldsymbol{r}_{j}-\boldsymbol{r}_{i}, r_{i j}=\left|\boldsymbol{r}_{i j}\right|$, and $\boldsymbol{n}_{i j}=\boldsymbol{r}_{i j}|| \boldsymbol{r}_{i j} \mid$. The repulsion parameter between water particles $\left(a_{i i}\right)$ is set at $25 k_{B} T$ for density $\rho=3$ to match the compressibility of liquid water at room temperature, if one DPD particle corresponds to one water molecule. In this study, as discussed later, the size of the water particle matches the volume of four $\mathrm{H}_{2} \mathrm{O}$ molecules, so we set $a_{i i}$ at 104 for water particles. Other repulsion parameters between particles of the same type are chosen to be the same value as the water particles. The repulsion parameters between particles of different types correspond to the mutual solubility, expressed as the Flory-Huggins $\chi$ parameter. In the case where the reduced density $\rho$ is 3 , this relation is as follows. ${ }^{19}$

$$
a_{i j}=a_{i i}+3.27 \chi_{i j}
$$

This equation was derived by applying the condition that density profiles of the binary immiscible system should be described correctly. We determined these parameters from atomistic simulation for each pair of particles of the different type. The mixing energies are calculated for a chemical structure equivalent to each component of DPD particles using an atomistic simulation by a Monte Carlo approach for pure components and their mixtures, and then the $\chi$-parameters are estimated. This procedure will be discussed below. The dissipative force $\boldsymbol{F}_{i j}^{D}$ is a hydrodynamic drag and is given by ${ }^{19}$

$$
\boldsymbol{F}_{i j}^{D}=\left\{\begin{array}{cc}
-\gamma \omega^{D}\left(r_{i j}\right)\left(\boldsymbol{n}_{i j} \cdot \boldsymbol{v}_{i j}\right) \boldsymbol{n}_{i j} & r_{i j}<r_{c} \\
0 & r_{i j} \geq r_{c}
\end{array}\right.
$$

where $\gamma$ is a friction parameter, $\omega^{D}\left(r_{i j}\right)$ is the weighting function, and $\boldsymbol{v}_{i j}=\boldsymbol{v}_{j}-\boldsymbol{v}_{i}$. The friction parameter is 
related to the viscosity of the system and acts in such a manner as to slow the relative velocity of two particles and to remove kinetic energy. The random force $\boldsymbol{F}_{i j}^{R}$ corresponds to thermal noise and is governed by the noise parameter $\sigma$ and a different weighting function $\omega^{R}\left(r_{i j}\right)$ as follows. ${ }^{19}$

$$
\boldsymbol{F}_{i j}^{R}=\left\{\begin{array}{cc}
\sigma \omega^{R}\left(r_{i j}\right) \zeta_{i j} \Delta t^{-1 / 2} \boldsymbol{n}_{i j} & r_{i j}<r_{c} \\
0 & r_{i j} \geq r_{c}
\end{array}\right.
$$

The randomness is contained in the element $\zeta_{i j}$, which is a randomly fluctuating variable with Gaussian statics.

$$
\begin{aligned}
& \left\langle\zeta_{i j}(t)\right\rangle=0 \\
& \left\langle\zeta_{i j}(t) \zeta_{k l}\left(t^{\prime}\right)\right\rangle=\left(\delta_{i k} \delta_{j l}+\delta_{i l} \delta_{j k}\right) \delta\left(t-t^{\prime}\right)
\end{aligned}
$$

They are uncorrelated for different pairs of particles and time. There is a relation between the two weighting functions and two parameters.

$$
\begin{aligned}
& \omega^{D}(r)=\left[\omega^{R}(r)\right]^{2} \\
& \sigma^{2}=2 \gamma k_{B} T
\end{aligned}
$$

In our simulation, we choose weighting functions as follows, ${ }^{19}$

$$
\omega^{D}\left(r_{i j}\right)=\left[\omega^{R}\left(r_{i j}\right)\right]^{2}=\left\{\begin{array}{cc}
\left(r_{c}-r_{i j}\right)^{2} & r_{i j}<r_{c} \\
0 & r_{i j} \geq r_{c}
\end{array}\right.
$$

The appearance of $\Delta t^{-1 / 2}$ in eq 7 is due to ensuring the consistent diffusion of particles independent of the stepsize of the integration as discussed by Groot and Warren. ${ }^{19,20}$ The spring force $\boldsymbol{F}_{i j}^{S}$ for a polymer is considered to be harmonic springs for the equilibrium bond distance $r_{s},{ }^{19}$

$$
\boldsymbol{F}_{i j}^{S}=-C\left(r_{s}-r_{i j}\right) \boldsymbol{n}_{i j}
$$

where $C$ is a spring constant.

In this study, we choose the interaction range $r_{c}$, the temperature $k_{\mathrm{B}} T$ and the particle mass $m$ as the units of length, energy and mass, respectively. The noise parameter $\sigma$ is set at 3.0 (the friction parameter $\gamma$ is 4.5). We set the bond distance at $r_{s}=0.86$ for the average distance of the nearest neighbor at a particle density $\rho=3$ and the spring constant at $C=100$ to obtain a very stiff chain for stretching. The simulated time is expressed in the unit of time $r_{c}\left(m / k_{\mathrm{B}} T\right)^{0.5}$, which corresponds to some tens of picoseconds. ${ }^{23}$ Time evolution of the system is calculated by a modified version of the velocity-Verlet algorithm ${ }^{19}$ at the empirical variable factor $\lambda=0.65$ with time steps of $\Delta t=0.05$. Our original DPD program has been coded and is used for following simulations.

\section{DPD Model for Nafion}

For reviewing the molecular structure of a Nafion polymer, an atomistic model of the monomer unit was

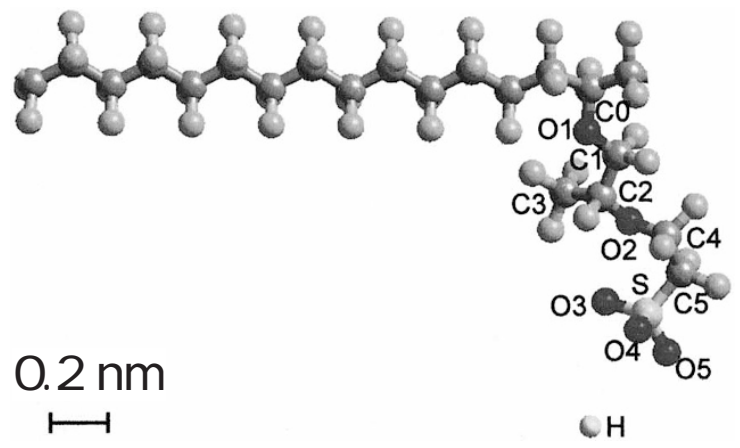

Figure 1. Molecular structure of a Nafion monomer. Several atoms are labeled to indicate their partial atomic charges in Table I.

Table I. Partial atomic charges of nafion

\begin{tabular}{lrlr}
\hline \multicolumn{1}{c}{ Atom } & Charge (amu) & \multicolumn{1}{c}{ Atom } & Charge (amu) \\
\hline C(backbone) & 0.552 & $\mathrm{~F}(\mathrm{C} 3)$ & -0.265 \\
F(backbone) & -0.273 & $\mathrm{O} 2$ & -0.512 \\
C0 & 0.455 & $\mathrm{C} 4$ & 0.774 \\
F(C0) & -0.273 & $\mathrm{~F}(\mathrm{C} 4)$ & -0.283 \\
O1 & -0.551 & $\mathrm{C} 5$ & 0.371 \\
C1 & 0.845 & $\mathrm{~F}(\mathrm{C} 5)$ & -0.267 \\
F(C1) & -0.256 & $\mathrm{~S}$ & 1.079 \\
C2 & 0.446 & $\mathrm{O} 3, \mathrm{O} 4, \mathrm{O} 5$ & -0.625 \\
F(C2) & -0.265 & $\mathrm{H}$ & 1.000 \\
C3 & 0.812 & & \\
\hline
\end{tabular}

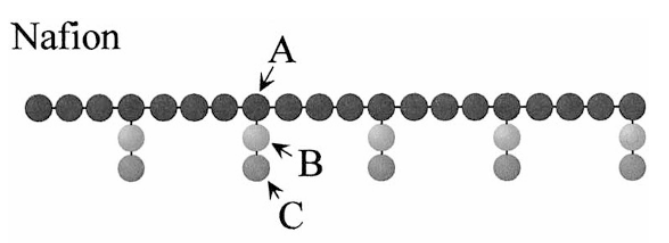

water<smiles>[18O]</smiles>

Figure 2. DPD particle model for a Nafion polymer and water. Particle size is approximately $0.61 \mathrm{~nm}$.

built using the Cerius ${ }^{2}$ software package from Accelrys Inc. Because most of the experimental studies of the hydrated Nafion membrane were performed for Nafion 1200 , we choose $n=7$ in the chemical structure of Nafion. Figure 1 shows a Nafion monomer structure. Several atoms are labeled to indicate partial atomic charges in Table I, discussed later. In the DPD method, a polymer is modeled by connecting spherical soft particles, which correspond to groups of several atoms. Referring to the atomistic model in Figure 1, the DPD model for the Nafion polymer is designed as shown in Figure 2. Particles A, B, and $\mathrm{C}$ approximately correspond to $-\mathrm{CF}_{2} \mathrm{CF}_{2} \mathrm{CF}_{2} \mathrm{CF}_{2}$,,$-\mathrm{OCF}_{2} \mathrm{C}\left(\mathrm{CF}_{3}\right) \mathrm{FO}-$, and $-\mathrm{CF}_{2} \mathrm{CF}_{2} \mathrm{SO}_{3} \mathrm{H}$, respectively. We find the volume of particle A is $0.12 \mathrm{~nm}^{3}$, the volume of particle $\mathrm{B}$ is $0.11 \mathrm{~nm}^{3}$, and the volume of particle $\mathrm{C}$ is $0.11 \mathrm{~nm}^{3}$. We can estimate that all particles have the same diameter of approximately $0.61 \mathrm{~nm}$ for spheres. In the actual syn- 
thesis process, an alcohol and water mixture is used for the solvent. However, for simplicity, only water is considered in this simulation. A water particle is modeled as the same size as the component of the Nafion polymer model. Because the volume of a $\mathrm{H}_{2} \mathrm{O}$ molecule is $0.03 \mathrm{~nm}^{3}$, the water particle $\mathrm{W}$ is to represent the same volume of $0.12 \mathrm{~nm}^{3}$ and four water molecules. Considering the simulated particle density $\rho r_{c}^{3}=3$, a cube of $r_{c}^{3}$ contains three particles and therefore corresponds to a volume of $0.36 \mathrm{~nm}^{3}$. Thus, the physical size of the unit of length $r_{c}$ must be $0.36^{1 / 3} \mathrm{~nm}=0.71 \mathrm{~nm}$ in the same treatment by Groot and Rabone. ${ }^{23}$

In this study, the number of repeating units in the Nafion polymer model has been chosen to be 5 , and the number of backbone segment is 20 . This is less than the actual Nafion polymer molecule; therefore, there is a concern for reproducibility of the polymeric property of the adopted DPD model. In the DPD simulation of block copolymer systems, phase diagram could be reproduced using a model polymer 10 segments. ${ }^{20}$ It is expected to reproduce the polymeric property in our simulation. In a preliminary simulation, however, we confirmed that the same mesoscopic structure as described in following sections could be obtained even if the number of repeating units is chosen to be 2 . Considering that the formation of the same mesoscopic structure is obtained at numbers of the repeating units of both 2 and 5, this Nafion polymer model has a sufficiently large number of repeating units to produce an equilibrium mesoscopic structure for this system.

\section{Interaction Parameter}

Interaction parameters for the conservative force between DPD particles are necessary for simulation. These parameters are related to the Flory-Huggins $\chi$ parameters as are indicated in eq 5. Several different computational methods are available for the estimation of $\chi$-parameters between polymer-polymer, polymersolvent, or solvent-solvent pairs. In the present study, we estimated the $\chi$-parameters from the mixing energy calculation using a Monte Carlo approach. ${ }^{26}$ The mixing energies are obtained by averaging a large number of configurations generated by the Monte Carlo approach for each pair of components. The $\chi$-parameters are estimated to be

$$
\chi=z\left(\frac{\Delta E_{12}}{R T}\right)
$$

where $z$ is the coordination number, i.e., the number of possible interaction partners. $\Delta E_{12}$ is the heat of mixing associated with pairwise interaction between components 1 and 2 , which is defined as

$$
\Delta E_{12}=E_{12}-\frac{1}{2}\left(E_{11}+E_{22}\right)
$$

Table II. Calculated $\chi$-parameters and corresponding repulsion parameters of DPD simulation for each pair of specific compositions $^{\mathrm{a}}$

\begin{tabular}{lcc}
\hline Pair & $\chi$ & $a_{i j}\left(k_{B} T\right)$ \\
\hline A-B & 0.022 & 104.1 \\
A-C & 3.11 & 114.2 \\
A-W & 5.79 & 122.9 \\
B-C & 1.37 & 108.5 \\
B-W & 4.90 & 120.0 \\
C-W & -2.79 & 94.9 \\
\hline${ }^{a}$ Compositions correspond to A: $-\mathrm{CF}_{2} \mathrm{CF}_{2} \mathrm{CF}_{2} \mathrm{CF}_{2}-$ \\
B:- $\mathrm{OCF}_{2} \mathrm{C}\left(\mathrm{CF}_{3}\right) \mathrm{FO}-, \mathrm{C}:-\mathrm{CF}_{2} \mathrm{CF}_{2} \mathrm{SO}_{3} \mathrm{H}$, and $\mathrm{W}: \mathrm{H}_{2} \mathrm{O}$.
\end{tabular}

where $E_{i j}$ is the energy of a particular $i j$ pair.

To ensure a high quality estimation of the mixing energy, we begin with a determination of partial atomic charges of the Nafion monomer and water using ab initio molecular orbital calculation with Gaussian98 software $^{31}$ at the B3LYP/6-31G(d) ${ }^{32,33}$ level of theory (Becke's three-parameter exchange functional with nonlocal correlation provided by the LYP expression). Calculated partial charges of Nafion are listed in Table I. The labels of the atoms are indicated in Figure 1. For partial charges of the water molecule, 0.424 is assigned for a hydrogen atom $(\mathrm{H})$ and -0.848 is assigned for an oxygen atom $(\mathrm{O})$. Atomistic simulations are then performed using Cerius ${ }^{2}$ for the estimation of the mixing energies by the Monte Carlo approach. Corresponding to DPD particles, the components used in this calculation are (A) $-\mathrm{CF}_{2} \mathrm{CF}_{2} \mathrm{CF}_{2} \mathrm{CF}_{2-}$, (B) $-\mathrm{OCF}_{2} \mathrm{C}\left(\mathrm{CF}_{3}\right) \mathrm{FO}-,(\mathrm{C})-\mathrm{CF}_{2} \mathrm{CF}_{2} \mathrm{SO}_{3} \mathrm{H}$, and (W) $\mathrm{H}_{2} \mathrm{O}$ after these structures are optimized by molecular mechanics.

The calculated $\chi$-parameters are listed in Table II corresponding to the conservative parameters for DPD. The diagonal terms of the conservative parameters are set at 104. Hydrophobic backbone particles (A) and side chain particles (B) are expected to be strongly immiscible with water particles (W). However the hydrophilic end particles of the side chain (C) are miscible with water particles (W) due to electrostatic interaction. Considering these repulsion parameters, we can speculate a microphase separation in a water-containing Nafion membrane. The interaction parameter based on the atomistic simulations depends on the adopted force field and evaluation methods. However, even if the magnitude of the interaction parameters listed in Table II slightly varies, a structure similar to the hydrated Nafion membrane as described in following sections could be obtained. This system is characterized by the nature of miscibility between water and the hydrophilic end particles of side chain and immiscibility between water and Nafion backbone particles. 


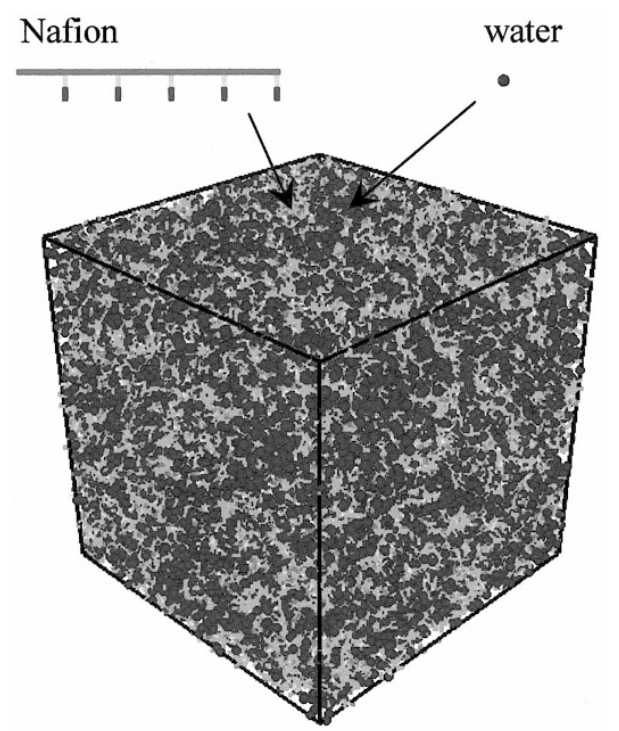

Figure 3. Initial condition of the $20 \mathrm{vol} \%$ hydrated Nafion membrane system. Nafion polymer particles are indicated by lines and water particles are drawn by spheres.

\section{Simulation Condition}

For the initial condition of DPD simulation, Nafion polymer particles and water particles are randomly dispersed into a unit cell of side length 40 , which is scaled by the unit of length $r_{c}$ and corresponds to about $28.4 \mathrm{~nm}$. Figure 3 shows an initial system for the $20 \mathrm{vol} \%$ water content. It contains 192000 DPD particles at density $\rho=3$. In this figure, the Nafion polymer skeletons are illustrated by flexible rods and water particles are drawn by spheres. Periodic boundary conditions are considered in all directions. According to the synthesis process of the Nafion membrane in the case of the casting procedure, this initial condition is reasonable with respect to a solution in an alcohol and water mixture at the initial stage of the experimental process. From this initial condition, the time evolution of DPD particles is calculated up to the equilibration, which is about $t=1000$, for water contents of 10,20 , and $30 \mathrm{vol} \%$.

\section{RESULTS AND DISCUSSION}

In Figure 4, the time dependent morphologies of a $20 \mathrm{vol} \%$ hydrated Nafion membrane are depicted. Nafion polymer particles are illustrated by lines and water particles are drawn by spheres in the same manner as in Figure 3. At the initial stage up to $t=10$, a small domain of water aggregation emerges. This domain developed into a large water cluster surrounded by hydrophilic end particles of the side chain of the Nafion polymer particles. The system reaches an equilibrium state around $t=1000$. Water particles and hydrophilic particles of the Nafion side chain form aggre-

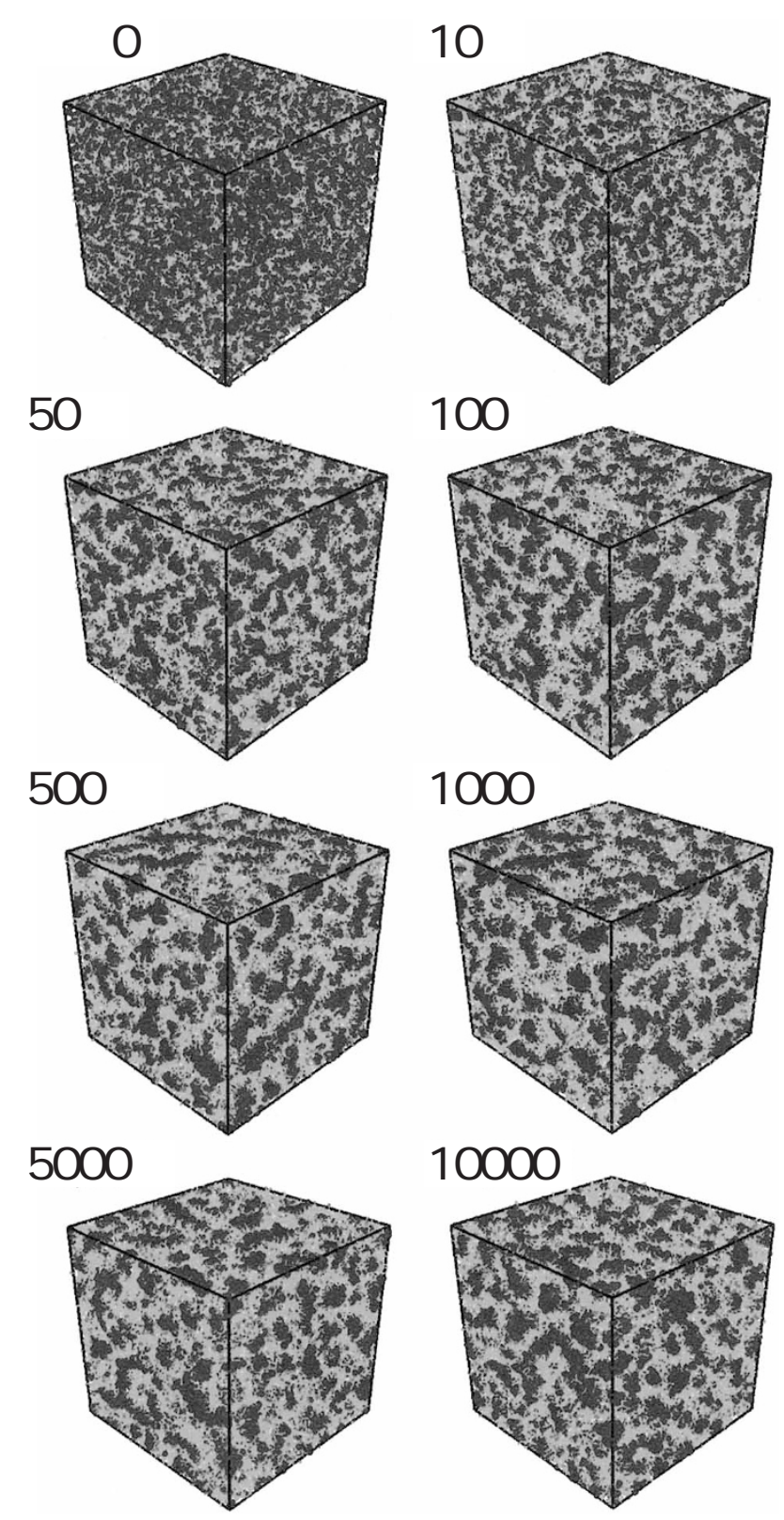

Figure 4. Time dependent morphologies of the $20 \mathrm{vol} \%$ hydrated Nafion membrane system. Nafion polymer particles are indicated by lines and water particles are drawn by spheres.

gates or clusters and are embedded in the hydrophobic phase of the Nafion backbone. We confirmed that the simulated structure is in equilibrium by checking the time evolution of the cluster size. The calculated radial distribution of water particles for the structure at $t=1000$ is shown in Figure 5. Sharp peaks at $r=0.86$ and $r=1.77$ originate from the first and second nearest water particles in a water cluster. As $r$ increases, these first large peaks decrease and the breaking point $g(r)=1$ is defined to be the end of the water cluster. A second peak is observed at $r=6.80$, which is based on the first nearest water cluster. A weak third peak is recognized at $r=12.45$, correlating to the second nearest water cluster. Fourier transformation of the radial 


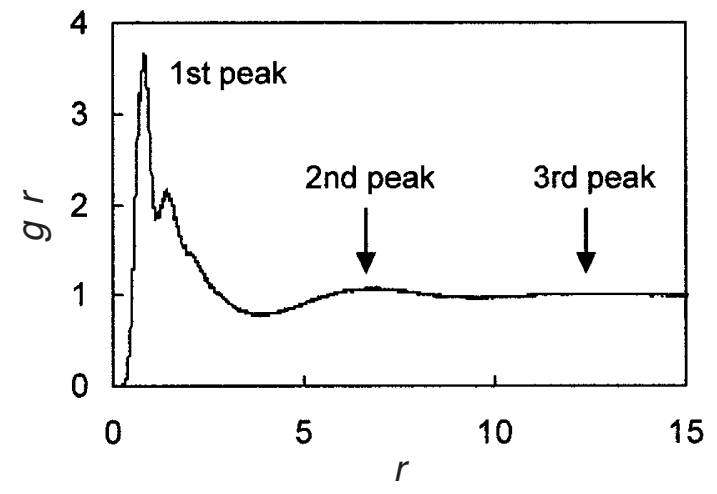

Figure 5. Radial distribution of water particles $g(r)$ for the $20 \mathrm{vol} \%$ hydrated Nafion membrane system at $t=1000$.

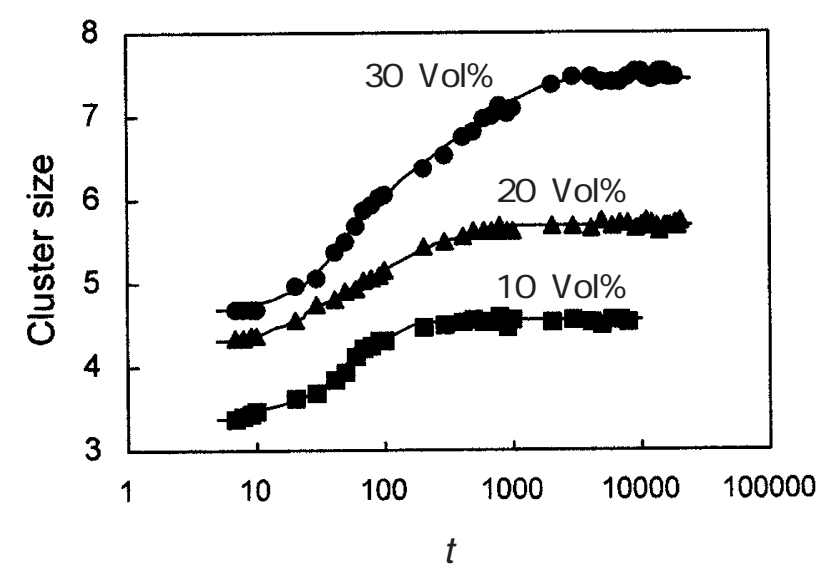

Figure 6. Time evolution of the cluster size scaled by the unit of length $r_{c}$ for the hydrated Nafion membrane system.

distribution data is related to the experimental observable pattern at small-angle X-ray scattering (discussed later). Figure 6 shows the cluster size as a function of time on a $\log$ scale. After small domains of water aggregation emerge at the initial stage, the cluster grows rapidly. Although the cluster growth slows down at the late stage, it is found to be in equilibrium around $t=500$ for $10 \mathrm{vol} \%, t=1000$ for $20 \mathrm{vol} \%$, and $t=5000$ for $30 \mathrm{vol} \%$. The scaling exponents of the structure growth are estimated as $0.07-0.16$. These values are small relative to the known domain growth of binary immiscible polymer systems. This is caused by the nature that the present system has a finite size of the structure similar to block copolymers, and is not fault of the simulation method. DPD simulations are successfully applied to investigate the domain growth of phase-separated immiscible polymers system. ${ }^{34}$

Corresponding small-angle X-ray scattering patterns are shown in Figure 7, which are obtained by Fourier transformation of the radial distribution data (e.g., Figure 5 for 20 vol\%). The shoulder peaks and their shift similar to the experimental work ${ }^{37}$ are recognized around $0.1-0.2 \mathrm{~nm}^{-1}$, though other peaks due to DPD particle size are observed at $1 \mathrm{~nm}^{-1}$.

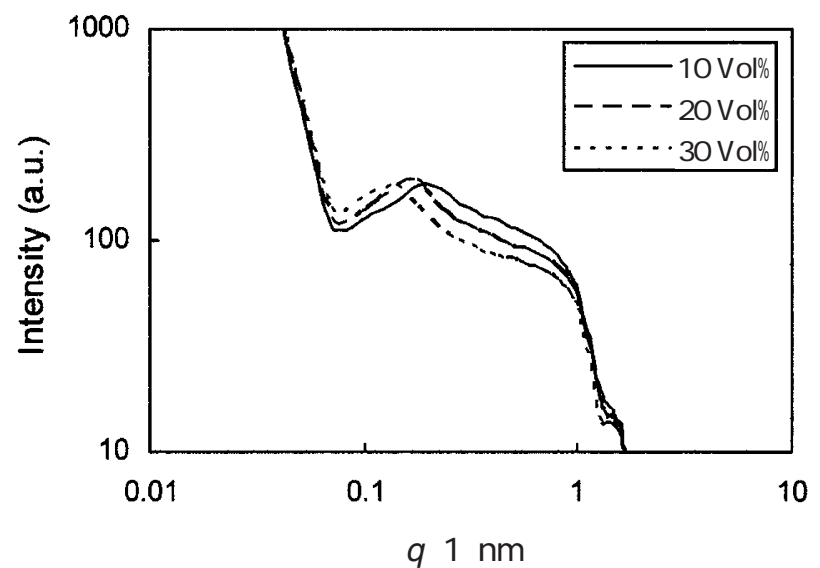

Figure 7. Calculated small-angle X-ray scattering patterns of equilibrium structure of the hydrated Nafion membrane. These data are obtained by Fourier transformation of the radial distribution data (e.g., Figure 5 for 20 vol\%).
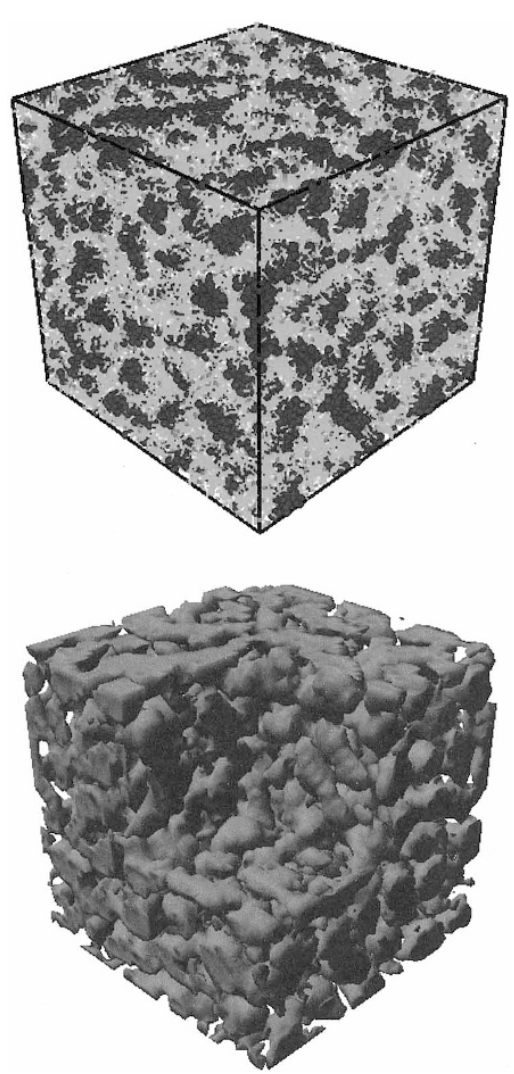

Figure 8. Equilibrium structure of the 20 vol $\%$ hydrated Nafion membrane system at $t=1000$. Illustrated in the same fashion as Figure 4 (top) and an isosurface representation of water particle regions including sulfonic acid groups at density $\rho=1.5$ (bottom).

Figure 8 shows the morphology at $t=1000$ by the same illustration in Figure 4 (top) and a surface of water particle regions including sulfonic acid groups (bottom), that is isosurface representation of particles $\mathrm{C}$ and $\mathrm{W}$ (level $\rho=1.5$ ). The isosurface image is partially cut so that an inside structure is clearly understood. Water particles and hydrophilic particles of the Nafion side chain form aggregates or clusters and are 

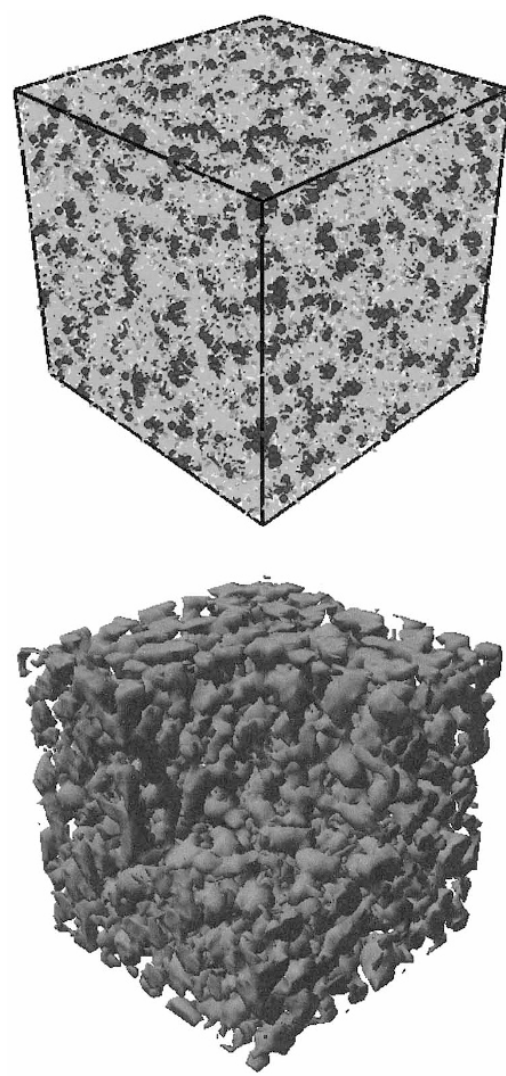

Figure 9. Equilibrium structure of $10 \mathrm{vol} \%$ hydrated Nafion membrane system at $t=1000$. Illustrated in the same fashion as Figure 4 (top) and an isosurface representation of water particle regions including sulfonic acid groups at density $\rho=1.5$ (bottom).

embedded in the hydrophobic phase of the Nafion backbone. Figures 9 and 10 show the equilibrium structure at $t=1000$ in the case of $10 \mathrm{vol} \%$ and at $t=5000$ in the case of $30 \mathrm{vol} \%$ water contents, respectively. Spongelike structures are similarly observed at these water contents.

Sponge-like or porous structures have been expected because of some experimental knowledge such as small-angle X-ray scattering studies. From a visual inspection of the simulated structure by rotating it in a graphics display, it is obvious that the shape of a water cluster is never spherical as proposed in the clusternetwork model ${ }^{1,5}$ but is an irregular shape. The regions of water particles are indistinguishable structures of water clusters and their connected channels. There are continuous paths in the water regions from any side of the cell to any other side of the cell. In a similar way for the Nafion backbone structure, this continuous path is also recognized. Therefore, it is percolating structure for components of both the Nafion backbone and water, similarly to a bicontinuous phase separation of the block copolymer systems. With careful inspection, however, we can notice that the characteristic shape of water regions, which consists of clusters and their connected channels, is somewhat unlike to the struc-
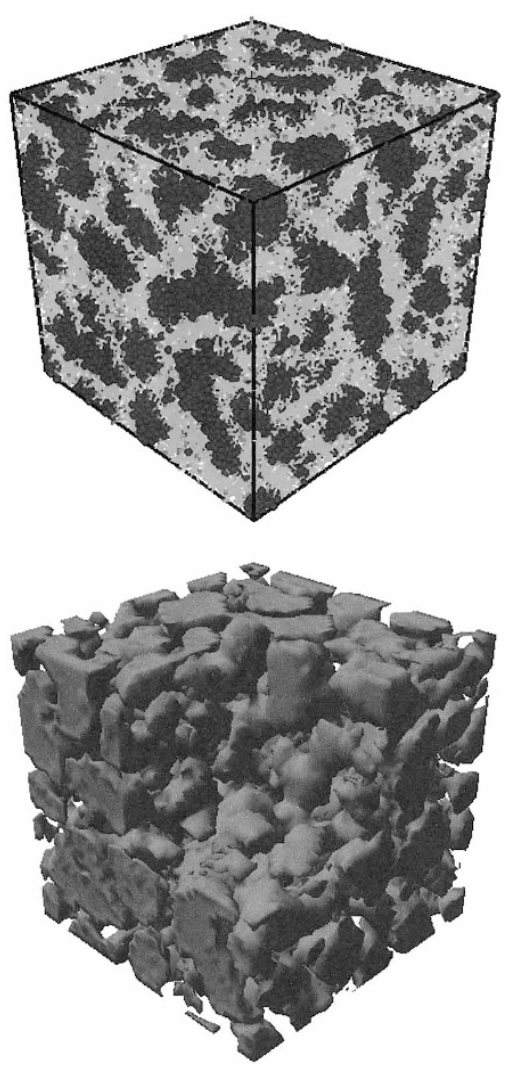

Figure 10. Equilibrium structure of $30 \mathrm{vol} \%$ hydrated Nafion membrane system at $t=5000$. Illustrated in the same fashion as Figure 4 (top) and an isosurface representation of water particle regions including sulfonic acid groups at density $\rho=1.5$ (bottom).

ture of block copolymer systems. There are several ways to verify a bicontinuous phase such as measurement of the Gaussian curvatures of the interface of the phase-separated bicontinuous structure. ${ }^{35}$ In this study, we confirmed the continuity of water regions by flow simulation using a lattice Boltzmann method. ${ }^{36}$ Complicated flow paths were observed in the water regions of hydrated Nafion membranes.

By comparing Figures $8-10$, it is clear that the cluster size of water is enlarged with the increase in water content. In previous experimental reports, though there are several proposed sizes of the hydrophilic aggregates, the average cluster size is estimated to be about $4 \mathrm{~nm}$, and its spacing is about $5 \mathrm{~nm}$ for hydrated Nafion membrane systems. ${ }^{1,3}$ To clarify the reliability of our simulation results, the size and spacing of the water clusters are examined from the equilibrium structures. The calculated data for cluster size and spacing are plotted as a function of water content in Figures 11 and 12, based on the radial distribution of water particles for the equilibrium structure at each water content. The corresponding actual scales are also indicated in the figures, estimated from the unit of length $r_{c}=0.71 \mathrm{~nm}$. As the water content increases for $10-30 \mathrm{vol} \%$, the cluster size changes to $3.2-5.3 \mathrm{~nm}$ and its spacing changes to $3.6-$ 


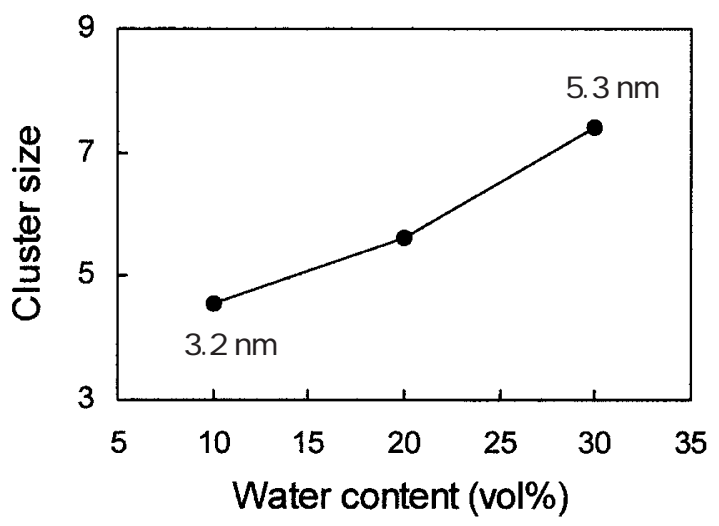

Figure 11. Dependence of the cluster size on the water content of the hydrated Nafion membrane. Cluster size is scaled by the unit of length $r_{c}$ and an actual scale is also indicated in the figure.

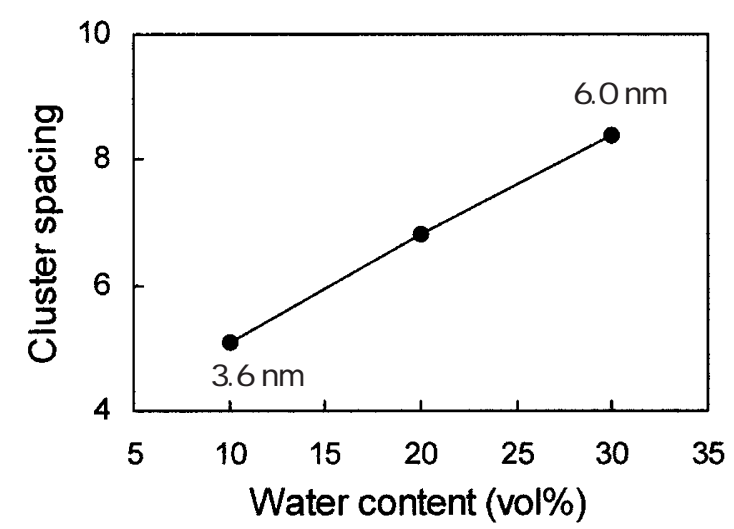

Figure 12. Dependence of the cluster spacing on the water content of the hydrated Nafion membrane. Cluster spacing is scaled by the unit of length $r_{c}$ and an actual scale is also indicated in the figure.

$6.0 \mathrm{~nm}$. These simulated scales of size and spacing are of reasonable magnitude with respect to the value of the experimental reports, and the dependence on water content is in good agreement with the previously reported experiments (cluster size of $3-5 \mathrm{~nm}$ ). ${ }^{1}$

From the above discussions, the simulated mesoscopic structure of the hydrated Nafion membranes is confirmed to be one of the reliable structures for actual materials. The simulated structures are essentially identical to the cluster-network model proposed from experimental studies; however, it is clarified that the structure is sponge-like or a bicontinuous microphase of the Nafion backbone and water, and the water regions are indistinguishable structures of water clusters and their channels. In previous analytical studies, the mechanical and transport properties of membranes have been discussed based on a model structure such as the cluster-network model, or by treating as averaged homogeneous substances. For example, the proton conductivity of the membrane is calculated based on the connectivity of clusters or percolation-type phenomena. ${ }^{5}$ Considering that the size and shape distributions of water clusters depend on the percolation threshold, more realistic properties can be predicted by introducing the mesoscopic structure obtained by this simulation. The simulated structure is also helpful in understanding the mechanism of proton transport inside the membrane.

In this study, we performed a modeling of DPD particles for a Nafion polymer and water and estimated their interaction parameters in cooperation with an atomistic simulation. This hierarchy procedure is one of the ways to bridge the gaps between different scale simulation methods. Although only a mesoscopic structure is simulated and discussed in this study, this method may have the possibility of being extended to the prediction of proton conductivity in a mesoscopic membrane model because DPD can directly treat the hydrodynamics, when we can model the behavior of the proton surrounded by water at a mesoscopic level for DPD simulation. Because DPD is a relatively new simulation method, some efforts need to be made to bridge the gap between atomistic and mesoscopic simulation, such as the determination of several parameters used in DPD simulation. Application of the mesoscopic simulation to actual materials will be investigated in the future work.

\section{CONCLUSIONS}

We have applied a mesoscopic simulation method, dissipative particle dynamics (DPD), to study the structure of hydrated Nafion membranes. In the DPD method, the dynamics among soft particles, which represent groups of several atoms, is simulated. Nafion polymer molecules are constructed by connecting soft DPD particles based on molecular structure. A water particle is also modeled as the same size particle as the Nafion polymer model. This means that the water particle represents a group of several $\mathrm{H}_{2} \mathrm{O}$ molecules. The repulsion parameter between DPD particles is related to the Flory-Huggins interaction parameter. They are estimated by an atomistic simulation calculating the mixing energies for pure components of DPD particles and their mixtures. The mixtures of Nafion polymer particles and water particles spontaneously form a sponge-like or porous structure. Water particles and hydrophilic particles of the Nafion side chain form aggregates or clusters and are embedded in the hydrophobic phase of the Nafion backbone. The bicontinuous structure of the Nafion backbone and water is recognized. It is an essentially identical but more realistic structure than some proposed models based on experimental studies. The size and spacing of water clusters are determined by calculating the radial distribution of wa- 
ter particles for equilibrium structures of the hydrated Nafion membranes for $10-30 \mathrm{vol} \%$ water content. The cluster size of the simulation model changes by 3.2 to $5.3 \mathrm{~nm}$ and its spacing changes by 3.6 to $6.0 \mathrm{~nm}$. These magnitudes of water clusters and their dependence on the water content are in agreement with experimental reports. The simulated mesoscopic structure is confirmed to be highly possible for hydrated Nafion membranes.

Acknowledgment. The authors would like to thank Dr. M. Kawasumi of Toyota Central R\&D Labs., Inc., for helpful discussions and useful comments.

\section{REFERENCES}

1. T. D. Gierke, G. E. Munn, and F. C. Wilson, J. Polym. Sci., Polym. Phys. Ed., 19, 1687 (1981).

2. T. D. Gierke and W. Y. Hsu, in "Perfluorinated Ionomer Membranes", A. Eisenberg and H. L. Yeager Eds., ACS Symposium Series No. 180, American Chemical Society, Washington, D.C., 1982, p 283.

3. W. Y. Hsu and T. D. Gierke, J. Membr. Sci., 13, 307 (1983).

4. K. A. Mauritz and C. E. Rogers, Macromolecules, 18, 483 (1985).

5. M. Eikerling, A. A. Kornyshev, and U. Stimming, J. Phys. Chem., 101, 10807 (1997).

6. S. Nemat-Nasser and J. Y. Li, J. Appl. Phys., 87, 3321 (2000).

7. P. A. Cirkel and T. Okada, Macromolecules, 33, 4921 (2000).

8. H.-G. Haubold, T. Vad, H. Jungbluth, and P. Hiller, Electrochim. Acta, 46, 1559 (2001).

9. M. Fujimura, T. Hashimoto, and H. Kawai, Macromolecules, 15, 136 (1982).

10. Z. Porat, J. R. Fryer, M. Huxham, and I. Rubinstein, J. Phys. Chem., 99, 4667 (1995).

11. T. Xue, J. S. Trent, and K. Osseo-Asare, J. Membr. Sci., 45, 261 (1989).

12. M. Ludvigsson, J. Lindgren, and J. Tegenfeldt, Electrochim. Acta, 45, 2267 (2000).

13. R. Buzzoni, S. Bordiga, G. Ricchiardi, G. Spot, and A. Zecchina, J. Phys. Chem., 99, 11937 (1995).

14. J. G. E. M. Fraaije, B. A. C. van Vlimmeren, N. M. Maurits, M. Postma, O. A. Evers, C. Hoffmann, P. Altevogt, and G. Goldbeck-Wood, J. Chem. Phys., 106, 4260 (1997).

15. R. Hasegawa and M. Doi, Macromolecules, 30, 3086 (1997).

16. P. J. Hoogerbrugge and J. M. V. A. Koelman, Europhys. Lett.,
19, 155 (1992).

17. J. M. V. A. Koelman and P. J. Hoogerbrugge, Europhys. Lett., 21, 363 (1993).

18. A. D. Mackie, J. B. Avalos, and V. Navas, Phys. Chem. Chem. Phys., 1, 2039 (1999).

19. R. D. Groot and P. B. Warren, J. Chem. Phys., 107, 4423 (1997).

20. R. D. Groot and T. J. Madden, J. Chem. Phys., 108, 8713 (1998).

21. C. M. Wijmans, B. Smit, and R. D. Groot, J. Chem. Phys., 114, 7644 (2001).

22. T. Spyriouni and C. Vergelati, Macromolecules, 34, 5306 (2001).

23. R. D. Groot and K. L. Rabone, Biophys. J., 81, 725 (2001).

24. B. E. Eichinger, D. R. Rigby, and M. H. Muir, Comp. Polym. Sci., 5, 147 (1995).

25. J. D. Honeycutt, Macromolecules, 27, 5377 (1994).

26. C. F. Fan, B. D. Olafson, M. Blanco, and S. L. Hsu, Macromolecules, 25, 3667 (1992).

27. S. J. Mumby, P. Sher, and B. E. Eichinger, Polymer, 34, 2540 (1993).

28. A. R. Tiller and B. Gorella, Polymer, 35, 3251 (1994).

29. F. H. Case and J. D. Honeycutt, TRIP, 2, 259 (1994).

30. L. A. Zook and J. Leddy, Anal. Chem., 68, 3793 (1996).

31. Gaussian 98, Revision A.7, M. J. Frisch, G. W. Trucks, H. B. Schlegel, G. E. Scuseria, M. A. Robb, J. R. Cheeseman, V. G. Zakrzewski, J. A. Montgomery, Jr., R. E. Stratmann, J. C. Burant, S. Dapprich, J. M. Millam, A. D. Daniels, K. N. Kudin, M. C. Strain, O. Farkas, J. Tomasi, V. Barone, M. Cossi, R. Cammi, B. Mennucci, C. Pomelli, C. Adamo, S. Clifford, J. Ochterski, G. A. Petersson, P. Y. Ayala, Q. Cui, K. Morokuma, D. K. Malick, A. D. Rabuck, K. Raghavachari, J. B. Foresman, J. Cioslowski, J. V. Ortiz, A. G. Baboul, B. B. Stefanov, G. Liu, A. Liashenko, P. Piskorz, I. Komaromi, R. Gomperts, R. L. Martin, D. J. Fox, T. Keith, M. A. Al-Laham, C. Y. Peng, A. Nanayakkara, C. Gonzalez, M. Challacombe, P. M. W. Gill, B. Johnson, W. Chen, M. W. Wong, J. L. Andres, C. Gonzalez, M. Head-Gordon, E. S. Replogle, and J. A. Pople, (Gaussian, Inc., Pittsburgh, 1998).

32. A. D. Becke, J. Chem. Phys., 98, 5648 (1993).

33. C. Lee, W. Yang, and R. G. Parr, Phys. Rev. B, 37, 785 (1988).

34. K. E. Novik and P. V. Coveney, Phys. Rev. E, 61, 435 (2000).

35. Y. Nishikawa, H. Jinnai, T. Koga, T. Hashimoto, and S. T. Hyde, Langmuir, 14, 1242 (1998).

36. H. Hayashi, S. Yamamoto, and S. Hyodo, Int. J. Mod. Phys. $B, \mathbf{1 7}, 135$ (2003).

37. G. Gebel, Polymer, 41, 5829 (2000). 